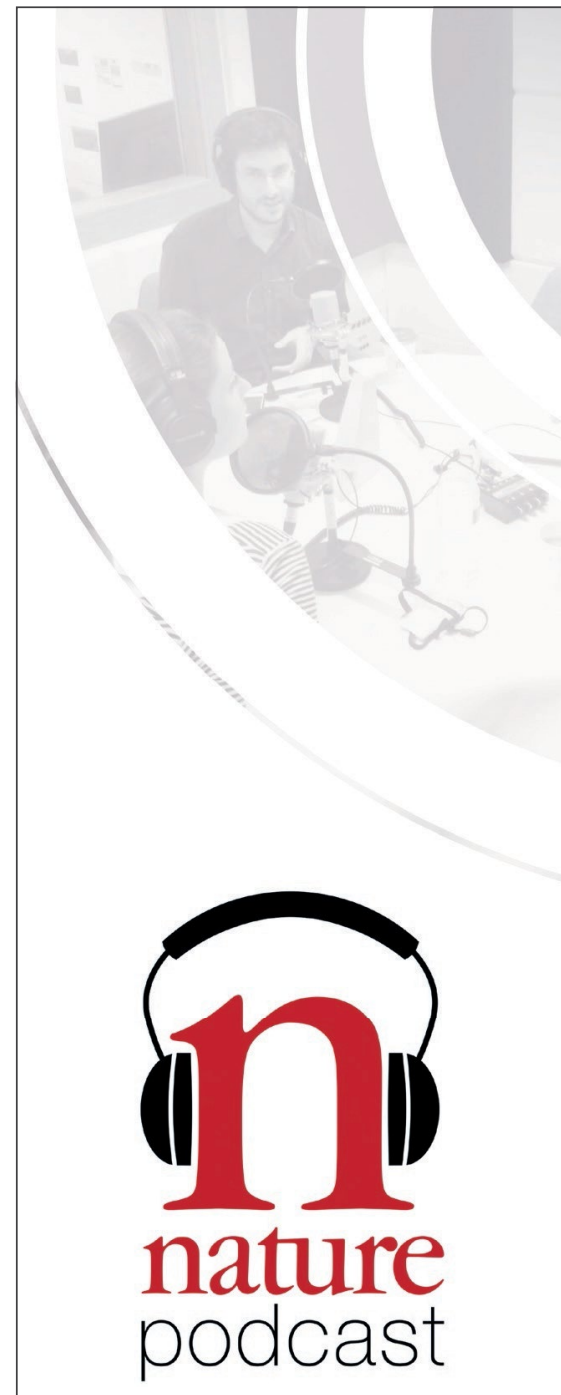

The week's best science, from the world's \#1 science journal.

NATURE.COM/NATURE/PODCAST

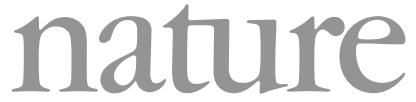

IMAGING

\section{Crikey! Croc MRIs}

Proc. R Soc. B 285: 20180178 (2018).

What happens when you stick a Nile crocodile in an MRI machine? You get a first look at sensory processing in a novel, poikilothermic animal.

Crocodiles are the closest living relatives to birds, which, despite the differences between the brains of avian models and humans, are often used in neurological research. What's shared between birds and crocodiles might provide some extra evolutionary insight. The anatomy of the crocodilian brain has was lacking, until researchers figured out how to immobilize and sedate juvenile Nile crocodiles for use in an MRI machine. With a customized procedure in place, they recorded blood oxygen level-dependent signal changes in the animals' telencephalon while they listened to Bach and were presented with different colors.

$E P N$ been characterized but functional information

\section{IMAGING}

Move freely with miniLFM

Nat. Methods https://doi.org/10.1038/s41592018-0008-0 (2018).

A new microscope from Alipasha Vaziri's lab at Rockefeller University in New York is taking volumetric neuronal image deeper into the brain of freely moving mice. The device, dubbed miniLFM, combines three previous technologies to enable the feat: light field microscopy, to capture volumetric data with minimal phototoxicity; a seeded iterative demixing (SID) computational strategy, to overcome modified miniscopes, to reduce the overall weight. Adult mice could carry the head-mounted devices without ill effect, and the researchers were able to record neuronal activity up to $360 \mu \mathrm{m}$ deep in the hippocampus of freely moving animals. light scattering in deeper tissue; and

\section{AUTISM SPECTRUM DISORDERS \\ A marker of sociality}

Sci. Transl. Med. 10, eaam9100 (2018).

Modeling disorders characterized by social impairments, like autism spectrum disorder (ASD), is a tricky task in animals that don't share many social behaviors with humans. Nonhuman primates often make better models than rodents, given their cognitive abilities and sociality. Among rhesus macaques, there can be extremes in social functioning within a group; new research uses these differences to identify a potential biomarker with translational relevance.

From legacy ethological data of male macaques housed at the California National Primate Research Center, the researchers sorted out a sample of extreme low-social animals from high; once validated, they tested cerebral spinal fluid for differences in several signaling molecules and pathways between animals in the two groups. One hormone in particular, arginine vasopressin (AVP), proved predictive. In an independent cohort of macaques and a small clinical sample, low levels of AVP were recorded in low-social macaques and male children with ASD. EPN

\section{DEVELOPMENTAL BIOLOGY}

\section{Epigenetic eyes}

Nat. Ecol. Evol. https://doi.org/10.1038/s41559018-0569-4 (2018).

Eyes aren't necessary to live in the dark, and many dark-adapted creatures have lost them entirely. Such is the case for morphs of Mexican tetras that have adapted to live in caves. For the first few days post-fertilization, cave tetras will develop eyes just like their sunlight-soaked river counterparts. Their eyes soon begin to degenerate though, and are completely absent in adults.

But unlike other blind species, there are no apparent genetic mutations driving the loss. Instead, the cause looks to be epigenetic. RNA sequencing revealed increased expression of $d n m t 3 b b .1$ in cavefish compared to river; this enzyme methylates DNA, which can modify gene expression without changing the DNA itself. Meanwhile, expression of genes involved with eye development was reduced. Chemically inhibiting the methylation process partially "rescued" the developing eyes that received the injection. 